\title{
SUBSETS CLOSE TO INVARIANT SUBSETS FOR GROUP ACTIONS
}

\author{
LEONID BRAILOVSKY, DMITRII V. PASECHNIK, AND CHERYL E. PRAEGER
}

(Communicated by Ronald Solomon)

\begin{abstract}
Let $G$ be a group acting on a set $\Omega$ and $k$ a non-negative integer. A subset (finite or infinite) $A \subseteq \Omega$ is called $k$-quasi-invariant if $\left|A^{g} \backslash A\right| \leq k$ for every $g \in G$. It is shown that if $A$ is $k$-quasi-invariant for $k \geq 1$, then there exists an invariant subset $\Gamma \subseteq \Omega$ such that $|A \Delta \Gamma|<2 e k\lceil(\ln 2 k)\rceil$. Information about $G$-orbit intersections with $A$ is obtained. In particular, the number $m$ of $G$-orbits which have non-empty intersection with $A$, but are not contained in $A$, is at most $2 k-1$. Certain other bounds on $|A \triangle \Gamma|$, in terms of both $m$ and $k$, are also obtained.
\end{abstract}

\section{INTRODUCTION}

Let $G$ be a group acting on a set $\Omega$, so that $\omega \mapsto \omega^{g}$ for $\omega \in \Omega, g \in G$. For a subset $A \subseteq \Omega$ we denote the image of $A$ under $g$ by $A^{g}=\left\{a^{g} \mid a \in A\right\}$. As usual, $A \triangle B$ will denote the symmetric difference of subsets $A, B \subseteq \Omega$.

For a subset $A$ of $\Omega$ we define

$$
k(A)=\max _{g \in G}\left|A^{g} \backslash A\right| .
$$

If $k=k(A)<\infty$, then $A$ is called a $k$-quasi-invariant subset. Thus a 0 -quasiinvariant subset is just a $G$-invariant (or invariant) subset, which is either an empty set or a union of $G$-orbits in $\Omega$. It was shown in [3] that a 1-quasiinvariant subset is either an invariant subset or an invariant subset with one point added or removed, and a classification of 2-quasi-invariant subsets was obtained in [4]. Note that every subset $A$ of $\Omega$ with $|A| \leq k$ is trivially $k$-quasi-invariant.

There are several places in the literature where results applicable to $k$-quasiinvariant subsets have been proved without a formal definition of $k$-quasiinvariance being given. For example, in [9] group actions for which every subset of $\Omega$ is $k$-quasi-invariant were considered, and bounds were obtained on the number and sizes of $G$-orbits. Here we consider the nature of a single $k$-quasiinvariant subset of $\Omega$. Also, if a $k$-quasi-invariant subset $A$ has cardinality greater than $k$, then the collection $\mathscr{A}=\left\{A^{g} \mid g \in G\right\}$ of images of $A$ under elements of $G$ forms a family of self-intersecting subsets of $\Omega$, or, more precisely, a $(|A|-k)$-intersecting family. (Note that, for $A^{g}, A^{h} \in \mathscr{A}$, we have

Received by the editors December 27, 1992.

1991 Mathematics Subject Classification. Primary 20B05, 20B07. 
$\left|A^{g} \cap A^{h}\right| \geq|A|-k \neq 0$.) Such families have been studied extensively (see for example [6] and [7]) mainly from the point of view of seeking bounds on $|\mathscr{A}|$. Normally these families have no group of permutations associated with them, as does our example. Our aim in this paper is to obtain upper bounds on the number of points by which a $k$-quasi-invariant subset may differ from its closest invariant subset.

Definition. Let $X$ be a subset of $\Omega$ and let $\left\{\Gamma_{i}\right\}_{i \in I}$ be the set of invariant subsets of $\Omega$. The invariant difference of $X$, denoted $d(X)$, is defined by

$$
d(X)=\min _{i \in I}\left(\left|X \triangle \Gamma_{i}\right|\right)
$$

Since the empty set is $G$-invariant, we always have $d(X) \leq|X|$. We prove the following. (Note that $\lceil x\rceil$ (resp. $\lfloor x\rfloor$ ) denotes the smallest (resp. the greatest) integer $y$ such that $y \geq x$ (resp. $x \geq y)$.)

Theorem A. Let $A$ be a $k$-quasi-invariant subset. Then $d(A)$ is finite and satisfies

$$
d(A)<2 e k\lceil(\ln 2 k)\rceil,
$$

where $e$ is the base of natural logarithms.

We do not know if this bound can be attained. However, if $G$ is transitive on $\Omega$, then we shall obtain in Corollary 2.6 a different bound, namely $d(A) \leq 2 k-$ 1 , and this bound is sharp. For example, it is attained whenever $G$ is a pointtransitive group of collineations of a $d$-dimensional projective space $P G(d, 2)$ over the two-element field $G F(2)$ and $A$ is a hyperplane. A better bound than that in Theorem $\mathrm{A}$, for $k \leq 31$, may be obtained combining Theorems $\mathrm{B}$ and C below.

We shall say that a $G$-orbit $\Omega_{i}$ has a proper intersection with a subset $A$ if $A \cap \Omega_{i}$ is non-empty and $\Omega_{i}$ is not contained in $A$.

Theorem B. Let $A$ be a $k$-quasi-invariant subset of $\Omega$. Then $A$ has proper intersections with at most $2 k-1$ orbits.

In what follows the number of $G$-orbits in $\Omega$ that intersect $A$ properly is denoted by $m(A)$; recall that $k(A)=\max _{g \in G}\left|A^{g} \backslash A\right|$.

The bound of Theorem B is sharp. It is attained, for example, when $G$ is an elementary abelian 2-group of rank $r, \Omega$ is the disjoint union of the coset spaces of all subgroups of $G$ of index 2 , and $A$ is a set consisting of one point from each orbit of $G$. Then $k(A)=k=2^{r-1}$ and $m(A)=2^{r}-1=2 k-1$.

It is also possible to derive a bound on $d(A)$ in terms of $m(A)$ and $k(A)$. The following result gives a much better bound than that in Theorem $\mathrm{A}$ in the case when $m(A)$ is relatively small with respect to $k$.

Theorem C. Let $A$ be a $k$-quasi-invariant subset of $\Omega$. Then

$$
d(A) \leq 2 k(3 / 2)^{\left\lceil\log _{2} m\right\rceil}-2^{\left\lceil\log _{2} m\right\rceil},
$$

where $m=m(A)$.

It is worth mentioning that the proof of Theorem $\mathrm{C}$ is based on Proposition 4.1, which is of some independent interest. The latter states that, given a decomposition $A=B_{1} \cup B_{2}$ such that the images of $B_{1}$ under the action of $G$ 
never intersect those of $B_{2}$, one has $k\left(B_{1}\right)+k\left(B_{2}\right) \leq 3 k / 2$, and this bound is sharp.

These results may be viewed as deductions about the action of a group $G$ from assumptions about the action of individual elements of $G$. From this point of view they bear a similarity to a recent result of G.M. Bergman and H.W. Lenstra [1] which deduces structural information about a group $G$ (acting on the space $[G: H]$ of cosets of a subgroup $H$ ) from assumptions about the action of a point stabilizer $H$. The result [1, Theorem 3] of Bergman and Jenstra will be used in a surprising way in Section 2 and we are grateful to Peter M. Neumann for showing us the proof of Theorem 2.4.

One crucial aspect of our proof is our use of a result of Birch, Burns, Oates Macdonald, and (P.M.) Neumann [2] (which was inspired by a result of B.H. Neumann [8]) to show the existence of finite $G$-orbits and to bound both their number and their length. The way we use it is as follows.

Remark 1. If $A$ is a finite $k$-quasi-invariant subset and $|A|=n>k$, then $A^{g} \cap A \neq \varnothing$ for every $g \in G$ and, by Theorem 2 of [2], there exists a $G$-orbit that has at most $n^{2}$ elements.

One of the results we prove in Section 2 provides a small improvement to Theorem 2 of [2] for transitive permutation groups.

Proposition D. Let $G$ be a transitive permutation group on a set $\Omega$ of size $|\Omega| \geq n^{2}$ and let $A$ be an $n$-element subset of $\Omega$. Then there is an element $g \in G$ such that $A \cap A^{g}=\varnothing$.

Theorem 2 of [2] implies this result when $|\Omega|>n^{2}$. Proposition D follows from Theorem 2.1 on noting that any $n$-element subset $A$ for which the conclusion of Proposition D fails is $(n-1)$-quasi-invariant.

Another more elementary fact, which helps in our analysis, is given in Remark 2 , the proof of which is straightforward.

Remark 2. Suppose that $A \subseteq \Gamma$, where $\Gamma$ is an invariant subset. Then $A$ is $k$-quasi-invariant if and only if $\Gamma \backslash A$ is $k$-quasi-invariant.

The rest of the paper is organized as follows. In Section 2 we will obtain bounds for the invariant difference of a $k$-quasi-invariant set in the case of a transitive group action. The general case will be considered in Section 3, where Theorems A and B will be proved. Section 4 contains a proof of Theorem $\mathrm{C}$.

\section{Transitive CASE}

In this section we will assume that $G$ acts transitively on $\Omega$.

Suppose first that $A$ is a finite $k$-quasi-invariant subset of $\Omega$. The following lemma gives an upper bound on the size of $\Omega$.

Theorem 2.1. Let $A$ be a finite $k$-quasi-invariant subset of cardinality $n>k$ for a transitive group $G$ on $\Omega$. Then

$$
|\Omega|<\frac{n^{2}}{n-k} .
$$

Proof. In view of Remark 1 , it follows that $|\Omega|<\infty$. Hence, we may assume that $G$ is finite, by considering the constituent of $G$ acting on $\Omega$ instead of $G$ if necessary. Fix some point $\omega \in \Omega$ and let $G_{\omega}$ denote the stabilizer of $\omega$ in $G$. Since the action of $G$ is transitive, we may write $A=\left\{\omega^{g_{1}}, \ldots, \omega^{g_{n}}\right\}$, 
where $g_{1}, \ldots, g_{n} \in G$. By (1), for every $g \in G$ there exist at least $n-k$ ordered pairs $\left(\omega^{g_{i}}, \omega^{g_{j}}\right) \in A \times A$ which satisfy

$$
\omega^{g_{i} g}=\omega^{g_{j}} .
$$

Also, there are some $g \in G$, for example the identity element, for which there are more than $n-k$ ordered pairs $\left(\omega^{g_{i}}, \omega^{g_{j}}\right) \in A \times A$ which satisfy the above condition. Note that $\omega^{g_{i} g}=\omega^{g_{j}}$ if and only if $g \in g_{i}^{-1} G_{\omega} g_{j}$. By counting the number of triples $(\alpha, \beta, g)$ with $\alpha, \beta \in A, g \in G$ and $\alpha^{g}=\beta$, we obtain

$$
|G|<\left|G_{\omega}\right| \cdot \frac{n^{2}}{n-k}
$$

whence inequality (2) follows.

It follows immediately that if a finite $k$-quasi-invariant subset $A$ is large enough relative to $k$, then $A$ differs from $\Omega$ by at most $k$ points. From (2) we obtain

$$
|\Omega \backslash A|<\frac{n^{2}}{n-k}-n=k+\frac{k^{2}}{n-k} .
$$

Corollary 2.2. Let $A$ be a finite $k$-quasi-invariant subset for a transitive group $G$ on $\Omega$, such that $|A|=n \geq k^{2}+k$. Then $|\Omega \backslash A| \leq k$.

Proof. The result follows immediately from (3).

The bound in Theorem 2.1 is nearly best possible. For example, $|\Omega|=\left\lfloor\frac{n^{2}}{n-k}\right\rfloor$ is attained whenever $k$ is a power of a prime: take $\Omega$ to be the points of the Desarguesian projective plane $P G_{2}(k), G$ any point-transitive group of collineations, and $A$ the complement of a line in $\Omega$.

Corollary 2.3. Let $G$ be transitive on a finite set $\Omega$ and let $A$ be a $k$-quasiinvariant subset of $\Omega$. Then $d(A) \leq 2 k-1$.

Proof. Since $G$ acts transitively on $\Omega$, we have $d(A)=\min (|A|,|\Omega \backslash A|)$. Suppose $|A| \geq 2 k$. Then, by (3), $|\Omega \backslash A|<2 k$, so $d(A) \leq 2 k-1$.

This bound is attained, for example, when $\Omega$ is the set of points of $P G(d, 2)$, the $d$-dimensional projective space over $G F(2)$, and $A$ is a hyperplane. In this case $n=|A|=2^{d-1}-1$ and $A$ is $k$-quasi-invariant for $k=2^{d-2}$. More generally, the bound is attained in the context of a certain generalization of projective geometry. An Hadamard design is a $2-(4 k-1,2 k-1, k-1)-$ design, that is a set $\Omega$ of $4 k-1$ points and a set of $(2 k-1)$-element subsets of $\Omega$, called blocks, such that each pair of points lies in $k-1$ blocks (see [5, p. 97]). Such a design has $4 k-1$ blocks, that is, it is symmetric, and, consequently each pair of blocks intersects in exactly $k-1$ points. Thus, if $A$ is a block, then $A$ is $k$-quasi-invariant for any group of automorphisms. It follows that, whenever there is a point-transitive Hadamard design, there is an example of a $k$-quasi-invariant set for which the bound of Corollary 2.3 is attained. Such designs exist, for example, if $4 k-1$ is an odd prime power (the Paley designs [5, p. 97]) or if $k$ is a power of 2 (the projective spaces already mentioned).

The following result, which was communicated to us by Peter M. Neumann, states that, if $A$ is an infinite $k$-quasi-invariant subset of $\Omega$, then $A$ is co-finite in $\Omega$. 
Theorem 2.4 (P.M. Neumann). Let $G$ act transitively on $\Omega$ and let $A$ be an infinite k-quasi-invariant subset of $\Omega$ for some positive integer $k$. Then $|\Omega \backslash A|<$ $\infty$.

Proof. Let $S$ be a finite non-abelian simple group and let $W$ be the restricted wreath product of $S$ by $G$ with respect to the action of $G$ on $\Omega$. Then $W$ is the semi-direct product of $K$ by $G$, where $K$ is the restricted direct product of copies of $S$ indexed by $\Omega$ and $G$ acts on $K$ by permuting its simple direct factors. Since $S$ is non-abelian simple and $G$ is transitive on $\Omega$, it follows that $K$ is a minimal normal subgroup of $W$.

Let $S^{\Omega}$ denote the direct product of $|\Omega|$ copies of $S$ (so that $K$ is the subgroup of $S^{\Omega}$ of elements of finite support), and, for any subset $B \subseteq \Omega$, let $S^{B}$ denote the subgroup of $S^{\Omega}$ of elements with support contained in $B$. Set $H=S^{A} \cap K$, so that $H$ is the subgroup of $K$ consisting of elements with finite support contained in $A$.

Since $H$ is normal in $K$, the conjugates of $H$ in $W$ are the subgroups of the form $S^{A^{8}} \cap K$ for $g \in G$. Since $A \subseteq \Omega$ is $k$-quasi-invariant, it follows that $\left[H: H \cap H^{w}\right] \leq|S|^{k}$ for all $w \in W$. By the result of G.M. Bergman and H.W. Lenstra mentioned in the introduction [1, Theorem 3] there exists a normal

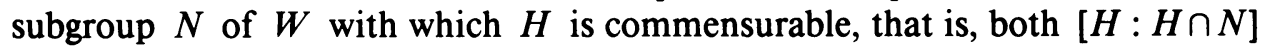
and $[N: H \cap N]$ are finite. Since $H \leq K, H \cap N=H \cap(N \cap K)$ and so $H$ is also commensurable with $N \cap K$ and we may, without loss of generality, assume that $N \leq K$. But $K$ is a minimal normal subgroup, so $N$ is trivial or $N=K$. If $N$ were the trivial group, then $H$ and hence $A$ would be finite, which would contradict our assumption. Hence $N=K$, but then $[K: H]<\infty$ and so $|\boldsymbol{\Omega} \backslash A|<\infty$.

For a transitive group $G$ on $\Omega$ the only invariant subsets are $\Omega$ and $\varnothing$, and hence for any $A \subseteq \Omega$ the invariant difference $d(A)=\min \{|A|,|\Omega \backslash A|\}$.

Corollary 2.5. Let $G$ act transitively on an infinite set $\Omega$ and let $A$ be $a k$ quasi-invariant subset of $\Omega$. Then $d(A) \leq k$.

Proof. Set $A^{\prime}=\Omega \backslash A$. By Remark $2, A^{\prime}$ is also $k$-quasi-invariant, and as $d(A)=d\left(A^{\prime}\right)$ we may assume that $d(A)=|A| \leq\left|A^{\prime}\right|$. It follows from Theorem 2.4 that $A$ is finite, and then by Remark 1 that $|A| \leq k$. Thus $d(A) \leq k$.

Combining Corollaries 2.3 and 2.5 we obtain

Corollary 2.6. Let $G$ be transitive on $\Omega$ and let $A$ be a k-quasi-invariant subset of $\Omega$. Then $d(A)=\min (|A|,|\Omega \backslash A|) \leq 2 k-1$.

\section{Bounds}

In this section we assume that the action of $G$ on $\Omega$ is not necessarily transitive. Also $A$ will denote a $k$-quasi-invariant subset of $\Omega$, either infinite or finite, with $|A|>k$. First we show that the $k$-quasi-invariance implies the following.

Lemma 3.1. $\left|A^{g} \backslash A\right|=\left|A \backslash A^{g}\right|$ for every $g \in G$.

Proof. When $A$ is finite $\left|A^{g} \backslash A\right|=\left|A^{g}\right|-\left|A \cap A^{g}\right|=|A|-\left|A \cap A^{g}\right|=\left|A \backslash A^{g}\right|$ for each $g \in G$. Thus we may assume that $A$ is infinite. Set $d_{g}=\left|A \backslash A^{g}\right|-\left|A^{g} \backslash A\right|$ 
for $g \in G$. Suppose there exists $g \in G$ such that our claim fails, that is $\left|A \backslash A^{g}\right|=n,\left|A^{g} \backslash A\right|=n-l, d_{g}=l \neq 0$. Without loss of generality we may assume that $d_{g}>0$, since we may interchange the roles of $A$ and $A^{g}$ and consider $g^{-1}$ instead of $g$. Translating by $g$, we have $\left|A^{g} \backslash A^{g^{2}}\right|=n$, $\left|A^{g^{2}} \backslash A^{g}\right|=n-l$. Hence

$$
m=\left|\left(A \cap A^{g}\right) \backslash A^{g^{2}}\right|=\left|A^{g} \backslash A^{g^{2}}\right|-\left|\left(A^{g} \backslash A\right) \backslash A^{g^{2}}\right| \geq\left|A^{g} \backslash A^{g^{2}}\right|-\left|A^{g} \backslash A\right|=l
$$

and

$$
\begin{aligned}
\left|\left(A^{g} \backslash A\right) \cap A^{g^{2}}\right| & =\left|A^{g} \backslash A\right|-\left|\left(A^{g} \backslash A\right) \backslash A^{g^{2}}\right| \\
& =(n-l)-\left(\left|A^{g} \backslash A^{g^{2}}\right|-\left|\left(A^{g} \backslash A^{g^{2}}\right) \cap A\right|\right) \\
& =(n-l)-(n-m)=m-l .
\end{aligned}
$$

Denote $x=\left|\left(A \backslash A^{g}\right) \cap A^{g^{2}}\right|, y=\left|A^{g^{2}} \backslash\left(A \cup A^{g}\right)\right|$. Since $x+y=\left|A^{g^{2}} \backslash A^{g}\right|=$ $n-l, y+m-l=\left|A^{g^{2}} \backslash A\right|$, we find that $\left|A^{g^{2}} \backslash A\right|=n-2 l+m-x$. Next, $\left|A \backslash A^{g^{2}}\right|=n+m-x$, and hence $d_{g^{2}}=2 l$.

A similar calculation yields $d_{g^{i}}=2^{i-1} l$ for any $i \geq 1$. Thus for a sufficiently large $i$ we will have $d_{g^{i}}>k$, which is a contradiction.

Next, we establish some notation. Let $\left\{\Omega_{i} \mid i \in I\right\}$ denote the set of $G$-orbits in $\Omega$. For $J \subseteq I$ set $\Omega_{J}=\bigcup_{j \in J} \Omega_{j}$ and $A_{J}=A \cap \Omega_{J}$. In particular, $A_{j}$ denotes $A \cap \Omega_{j}$.

If $A_{j}$ is not empty we will call $A_{j}$ an orbit segment of $A$. It turns out that the invariant difference of a set with a finite number of orbit segments has an additive property.

Lemma 3.2. Let $J$ be a finite subset of $I$. Then $d\left(A_{J}\right)=\left|A_{J} \Delta \Omega_{J^{\prime}}\right|$, where

$$
J^{\prime}=\left\{j \in J|| \Omega_{j} \backslash A|<| A_{j} \mid\right\} .
$$

In particular, $d\left(A_{J}\right)$ is finite and $d\left(A_{J}\right)=\sum_{j \in J} d\left(A_{j}\right)$.

Proof. The proof is by induction on $|J|$. The result is trivially true if $J=\varnothing$, and if $J=\{j\}$, then $d\left(A_{J}\right)=d\left(A_{j}\right)=\min \left(\left|A_{j}\right|,\left|\Omega_{j} \backslash A\right|\right)$ which by the definition of $J^{\prime}$ is equal to $\left|A_{J} \Delta \Omega_{J^{\prime}}\right|$, and by Corollary 2.6 is finite.

Assume then that $|J|>1$ and the result is true for smaller subsets. Let $j \in J$, and let $L=J \backslash\{j\}$ and $L^{\prime}=\left\{l \in L|| \Omega_{l} \backslash A|<| A_{l} \mid\right\}$. By induction $d\left(A_{L}\right)=\left|A_{L} \Delta \Omega_{L^{\prime}}\right|=\sum_{l \in L} d\left(A_{l}\right)<\infty$. Let $\Gamma$ be an invariant set. If $\Omega_{j} \nsubseteq \Gamma$, then $A_{J} \triangle \Gamma=A_{j} \cup\left(A_{L} \triangle \Gamma\right)$, while if $\Omega_{j} \subseteq \Gamma$, then $A_{J} \triangle \Gamma=\left(A_{j} \triangle \Omega_{j}\right) \cup\left(A_{L} \triangle\right.$ $\left(\Gamma \backslash \Omega_{j}\right)$ ). By Corollary 2.6, $A_{j} \triangle \Omega_{j}$ is finite, (and of course $\left.\left|A_{j} \Delta \Omega_{j}\right| \leq\left|A_{j}\right|\right)$. Then since $d\left(A_{L}\right)$ is finite, it follows that the minimum value $d\left(A_{J}\right)$ of $\left|A_{J} \triangle \Gamma\right|$ over all invariant sets $\Gamma$ is $\left|A_{j} \Delta \Omega_{j}\right|+\left|A_{L} \Delta \Omega_{L^{\prime}}\right|$. Thus $d\left(A_{J}\right)$ is finite and is equal to $\left|A_{J} \triangle \Omega_{J^{\prime}}\right|$, and to

$$
\left|A_{j} \Delta \Omega_{j}\right|+d\left(A_{L}\right)=d\left(A_{j}\right)+\sum_{l \in L} d\left(A_{l}\right) .
$$

Thus the lemma is proved.

To obtain bounds on $d(A)$, and on the number of $G$-orbits having a proper intersection with $A$, we decompose the set $\left\{\Omega_{i}\right\}_{i \in I}$ of all $G$-orbits in $\Omega$ in the following way. Let

$$
I=I_{0} \cup I_{1} \cup I_{\text {inf }} \cup I_{\text {fin }},
$$


where:

$$
\begin{aligned}
& i \in I_{0} \Leftrightarrow A_{i}=\varnothing ; \\
& i \in I_{1} \Leftrightarrow A_{i}=\Omega_{i} ; \\
& i \in I_{\text {inf }} \Leftrightarrow \Omega_{i} \text { is infinite and } \varnothing \neq A_{i} \neq \Omega_{i} ; \\
& i \in I_{\text {fin }} \Leftrightarrow\left|\Omega_{i}\right|<\infty, \varnothing \neq A_{i} \neq \Omega_{i} .
\end{aligned}
$$

For simplicity denote $A_{\text {inf }}:=A_{I_{\text {inf }}}$ and $A_{\text {fin }}:=A_{I_{\text {fin }}}$. Then $A=A_{I_{0}} \cup A_{I_{1}} \cup$ $A_{\text {inf }} \cup A_{\text {fin }}$. Let $\Gamma$ be a $G$-invariant subset of $\Omega$ such that $d(A)=|A \Delta \Gamma|$. Then $\Omega_{I_{1}} \subseteq \Gamma$ and $\Omega_{i} \cap \Gamma=\varnothing$ for any $i \in I_{0}$. Thus, we shall consider only those $G$-orbits that belong to $I_{\text {inf }} \cup I_{\text {fin }}$, i.e., have a proper intersection with $A$.

The following lemma will be used often to reduce from infinite to finite $k$ quasi-invariant subsets.

Lemma 3.3. Let $J$ be a finite subset of $I_{\mathrm{inf}} \cup I_{\mathrm{fin}}$ and let $A_{J}=\bigcup_{i \in J} A_{i}$ be the decomposition of $A_{J}$ into the disjoint union of the orbit segments in $\Omega_{J}$. For $j \in J$ denote $A_{j}^{\prime}=\Omega_{j} \backslash A_{j}$. Then:

(a) $B=\left(\bigcup_{i \in(J \backslash\{j\})} A_{i}\right) \cup A_{j}^{\prime}$ is a k-quasi-invariant subset.

(b) $d(B)=d\left(A_{J}\right)$.

Proof. Note that

$$
\left|B^{g} \backslash B\right|=\sum_{i \in(J \backslash\{j\})}\left|A_{i}^{g} \backslash A_{i}\right|+\left|\left(A_{j}^{\prime}\right)^{g} \backslash A_{j}^{\prime}\right| .
$$

Since $\left(A_{j}^{\prime}\right)^{g} \backslash A_{j}^{\prime}=A_{j} \backslash A_{j}^{g}$, it follows by Lemma 3.1 that

$$
\left|B^{g} \backslash B\right|=\sum_{i \in(J \backslash\{j\})}\left|A_{i}^{g} \backslash A_{i}\right|+\left|A_{j}^{g} \backslash A_{j}\right|=\left|A_{J}^{g} \backslash A_{J}\right| .
$$

Since $A_{J}$ is $k$-quasi-invariant, it follows that $B$ is $k$-quasi-invariant.

By Lemma 3.2, $d\left(A_{J}\right)=\sum_{i \in J} d\left(A_{i}\right)$ and $d\left(A_{j}\right)=\min \left(\left|A_{j}\right|,\left|\Omega \backslash A_{j}\right|\right)=$ $d\left(A_{j}^{\prime}\right)$, and part (b) follows.

Consider now $A_{\text {inf }}$, the part of $A$ which intersects properly with infinite $G$-orbits.

Lemma 3.4. (a) $\left|I_{\text {inf }}\right| \leq k$.

(b) $d\left(A_{\text {inf }}\right) \leq k$.

Proof. (a) Suppose $I_{\text {inf }}$ is non-empty and consider $A_{I_{\text {inf }}}$. Clearly, $A_{I_{\text {inf }}}$ is $k$ quasi-invariant. If $A_{I_{\text {inf }}}$ is finite, then $\left|A_{I_{\text {inf }}}\right| \leq k$, since otherwise, in view of Remark 1 , there would be $i \in I_{\text {inf }}$, such that $\Omega_{i}$ is finite, which is a contradiction. If $\left|A_{I_{\text {inf }}}\right| \leq k$, then, obviously, $\left|I_{\text {inf }}\right| \leq k$.

Assume now that $A_{I_{\text {inf }}}$ is infinite, and suppose, by way of contradiction, that $\left|I_{\text {inf }}\right|>k$. Let $J \subseteq I_{\text {inf }},|J|=k+1$. Clearly, $A_{J}$ is $k$-quasi-invariant. For every $j \in J$ define the set $B_{j}$ by

$$
B_{j}= \begin{cases}A_{j} & \text { if } A_{j} \text { is finite } \\ \Omega_{j} \backslash A_{j} & \text { otherwise. }\end{cases}
$$

Set $B=\bigcup_{j \in J} B_{j}$. By Lemma 3.3 (a), the set $B$ is $k$-quasi-invariant. On the other hand, by Theorem $2.4, B_{j}$ is finite for every $j \in J$, so $B$ is finite. Also, $B$ contains $k+1$ orbit segments, whence $|B|>k$. By Remark 1, we obtain a contradiction as above, which proves part (a). 
(b) To prove part (b) we apply the same arguments again. If $A_{I_{\text {inf }}}$ is finite, then $\left|A_{I_{\text {inf }}}\right| \leq k$, whence $d\left(A_{I_{\text {inf }}}\right) \leq k$. If $A_{I_{\text {inf }}}$ is infinite, then interchange each infinite orbit segment of $A_{I_{\text {inf }}}$ with its complement, as in part (a) and apply Lemma 3.3 (b).

We shall obtain now a bound for the number of finite $G$-orbits which intersect properly with $A$.

Lemma 3.5. $\left|I_{\text {fin }}\right| \leq 2 k\left(A_{\text {fin }}\right)-1 \leq 2 k-1$.

Proof. For any finite subset $J$ of $I_{\mathrm{fin}}$, the set $A_{J}$ is $k\left(A_{J}\right)$-quasi-invariant for the action of $G$ on $\Omega_{J}$, and $k\left(A_{J}\right) \leq k$. It is sufficient to prove that $|J| \leq 2 k\left(A_{J}\right)-1$, that is, it is sufficient to prove the lemma in the case where $I=I_{\text {fin }}$ is finite. Thus we assume that $I=I_{f i n}$ is finite.

Denote $s=|I|$. Let $H_{i}$ be the setwise stabilizer of $A_{i}$ in $G$ for $i \in I$. Consider the set $X$ of all pairs $(x, i)$, where $i \in I$ and $x \in H_{i}$. Since $A$ is $k$-quasi-invariant, every element of $G$ must stabilize at least $s-k$ of the orbit segments $A_{i}, i \in I$, so we have $|X|>|G|(s-k)$. The inequality is strict, since the identity element of $G$, for example, stabilizes all the orbit segments. On the other hand, $|X|=\sum_{i \in I}\left|H_{i}\right|$. Since $\frac{|G|}{\left|H_{i}\right|} \geq 2$ for every $i \in I$, we have

$$
s-k<\sum_{i \in I} \frac{\left|H_{i}\right|}{|G|} \leq \frac{s}{2},
$$

whence $|I|=s<2 k$.

Curiously, knowing the bound of Lemma 3.5, we can use it to show that this bound holds for the number of all $G$-orbits, finite and infinite, which intersect $A$ properly. This is the content of Theorem B. Before proving it, however, we obtain an upper bound on the movement $k\left(A_{\mathrm{fin}}\right)$ of $A_{\mathrm{fin}}$.

Lemma 3.6. $k\left(A_{\text {fin }}\right)=k-d\left(A_{\text {inf }}\right)$.

Proof. Let $\Gamma_{\text {inf }}$ be a $G$-invariant subset of $\Omega$ such that $d\left(A_{\text {inf }}\right)=\left|A_{\text {inf }} \Delta \Gamma_{\text {inf }}\right|$, and let $B=A_{\text {inf }} \Delta \Gamma_{\text {inf }}$. By Lemmas 3.3 and 3.2, we have $d(B)=d\left(A_{\text {inf }}\right)$. Choose $g_{1} \in G$ such that $k\left(A_{\mathrm{fin}}\right)=\left|A_{\mathrm{fin}}^{g_{1}} \backslash A_{\mathrm{fin}}\right|$ is maximal. Let $K$ denote the kernel of the action of $G$ on $\Omega_{I_{\text {fin }}}$. By Lemma 3.5, $\left|\Omega_{I_{\text {fin }}}\right|$ is finite, so $K$ has finite index in $G$. Hence all $K$-orbits in $\Omega_{I_{\text {inf }}}$ are infinite and, by Theorem 2 of [2], there exists $g_{0} \in K$ such that $B^{g_{1} g_{0}} \cap B=\varnothing$. Set $g=g_{1} g_{0}$. Then $\left(A_{\mathrm{fin}} \cup B\right)^{g}=A_{\mathrm{fin}}^{g_{1}} \cup B^{g}$. Since by Lemmas 3.4 and 3.5 the set $I_{\mathrm{fin}} \cup I_{\text {inf }}$ is finite, it follows from Lemma 3.3 (a) that

$$
\begin{gathered}
\left|\left(A_{\mathrm{fin}} \cup B\right)^{g} \backslash\left(A_{\mathrm{fin}} \cup B\right)\right|=\left|A_{\mathrm{fin}}^{g_{1}} \backslash A_{\mathrm{fin}}\right|+\left|B^{g} \backslash B\right| \leq k, \\
\text { so }\left|A_{\mathrm{fin}}^{g_{1}} \backslash A_{\mathrm{fin}}\right| \leq k-d(B)=k-d\left(A_{\mathrm{inf}}\right) \text {. }
\end{gathered}
$$

We are ready now to prove Theorem $\mathrm{B}$.

Proof of Theorem B. By Lemma 3.5, $\left|I_{\text {fin }}\right| \leq 2 k\left(A_{\text {fin }}\right)-1$. Also by Lemma 3.4, $I_{\text {inf }}$ is finite and it follows from Lemma 3.2 that $\left|I_{\text {inf }}\right| \leq d\left(A_{\text {inf }}\right)$. Thus, using also Lemma 3.6, we have

$$
\left|I_{\text {fin }}\right|+\left|I_{\text {inf }}\right| \leq\left(2 k\left(A_{\text {fin }}\right)-1\right)+d\left(A_{\text {inf }}\right)=2 k-1-d\left(A_{\text {inf }}\right) \leq 2 k-1 .
$$

Our next step is to obtain an upper bound for $d\left(A_{\text {fin }}\right)$. In fact, we can obtain a rough estimate of $d\left(A_{\text {fin }}\right)$ immediately from Lemma 3.5 and Corollary 2.6:

$$
d\left(A_{\text {fin }}\right) \leq(2 k-1)^{2} \text {. }
$$


In order to obtain a finer bound on $d\left(A_{\text {fin }}\right)$ we need the following technical lemma.

Lemma 3.7. Let $J \subseteq I_{\text {fin }}$ be a subset of $I_{\text {fin }}$, such that $\left|A_{J}\right|>k$. Then

$$
\sum_{j \in J} \frac{\left|A_{j}\right| \cdot\left(\left|\Omega_{j}\right|-\left|A_{j}\right|\right)}{\left|\Omega_{j}\right|}<k\left(A_{J}\right) \leq k .
$$

Proof. Fix an element $\omega_{j} \in \Omega_{j}$ for each $j \in J$. Let $H_{\omega_{j}}$ be the stabilizer of $\omega_{j}$ in $G$ and let $T_{j}$ be a transversal for $H_{\omega_{j}}$ in $G$.

Consider the set $X$ of all triples $\left(g, g_{1}, g_{2}\right)$, where $g \in G$ and $g_{1}, g_{2} \in T_{j}$, for some $j \in J$, are such that $\omega_{j}^{g_{1}}, \omega_{j}^{g_{2}} \in A_{j}$ and $\omega_{j}^{g_{1} g}=\omega_{j}^{g_{2}}$.

Note that $\omega_{j}^{g_{1} g}=\omega_{j}^{g_{2}} \Leftrightarrow g_{1} g g_{2}^{-1} \in H_{\omega_{j}}$. Hence

$$
|X|=\sum_{g \in G}\left|A_{J}^{g} \cap A_{J}\right|=\sum_{j \in J}\left|A_{j}\right|^{2} \cdot\left|H_{\omega_{j}}\right|=\sum_{j \in J} \frac{\left|A_{j}\right|^{2} \cdot|G|}{\left|\Omega_{j}\right|} .
$$

Since $A_{J}$ is a $k\left(A_{J}\right)$-quasi-invariant subset, so $\left|A_{J}^{g} \cap A_{J}\right| \geq\left(\left|A_{J}\right|-k\left(A_{J}\right)\right)$ for each $g \in G$. Also, for some $g \in G$, for example for the identity element, we have $\left|A_{J}^{g} \cap A_{J}\right|>\left(\left|A_{J}\right|-k\left(A_{J}\right)\right)$, whence

$$
\left|A_{J}\right|-k\left(A_{J}\right)<\sum_{j \in J} \frac{\left|A_{j}\right|^{2}}{\left|\Omega_{j}\right|}
$$

It follows that

$$
\sum_{j \in J} \frac{\left|A_{j}\right| \cdot\left(\left|\Omega_{j}\right|-\left|A_{j}\right|\right)}{\left|\Omega_{j}\right|}=\left|A_{J}\right|-\sum_{j \in J} \frac{\left|A_{j}\right|^{2}}{\left|\Omega_{j}\right|}<k\left(A_{J}\right) \leq k .
$$

Define now

$$
J_{x}=\left\{i \in I_{\text {fin }} \mid d\left(A_{i}\right) \geq \frac{k}{x}\right\},
$$

where $x$ is a positive real number.

Lemma 3.8. $r_{x}=d_{\text {def }}\left|J_{x}\right|<2 x$.

Proof. Let $i \in J_{x}$. Then $d\left(A_{i}\right) \geq \frac{k}{x}$, that is,

$$
\frac{k}{x} \leq\left|A_{i}\right| \leq\left|\Omega_{i}\right|-\frac{k}{x} \text {. }
$$

If $\left|A_{J_{x}}\right| \leq k$, then since $\left|A_{J_{x}}\right| \geq \frac{r_{x} k}{x}$, we have $r_{x} \leq x<2 x$ and the result is true. Hence we may assume that $\left|A_{J_{x}}\right|>k$ and, by Lemma 3.7 and using (4) (and noting that $y\left(\left|\Omega_{j}\right|-y\right) \geq \frac{k}{x}\left(\left|\Omega_{j}\right|-\frac{k}{x}\right)$ for $y \in\left[\frac{k}{x},\left|\Omega_{j}\right|-\frac{k}{x}\right]$ ), we obtain

$$
k>\sum_{j \in J_{x}} \frac{\left|A_{j}\right| \cdot\left(\left|\boldsymbol{\Omega}_{j}\right|-\left|A_{j}\right|\right)}{\left|\boldsymbol{\Omega}_{j}\right|} \geq \sum_{j \in J_{x}} \frac{\frac{k}{x}\left(\left|\boldsymbol{\Omega}_{j}\right|-\frac{k}{x}\right)}{\left|\boldsymbol{\Omega}_{j}\right|}=k \sum_{j \in J_{x}}\left(\frac{1}{x}-\frac{k}{x^{2}\left|\boldsymbol{\Omega}_{j}\right|}\right) .
$$

Hence

$$
1>\frac{r_{x}}{x}--\sum_{j \in J_{x}} \frac{k}{x^{2}\left|\Omega_{j}\right|}
$$


Since by (4), $\left|\Omega_{j}\right| \geq \frac{2 k}{x}$, we obtain

$$
\frac{r_{x}}{x}-1<\sum_{j \in J_{x}} \frac{k}{x^{2}\left|\Omega_{j}\right|} \leq \sum_{j \in J_{x}} \frac{k x}{2 k x^{2}}=\frac{r_{x}}{2 x},
$$

and the lemma follows.

By Corollary 2.6, we know that $d\left(A_{i}\right) \leq 2 k-1$ for each $i \in I_{\text {fin }}$. Taking $x=\frac{k}{2 k-1}$ in the statement of Lemma 3.8, we obtain

Corollary 3.9. There is at most one finite G-orbit $\Omega_{i}$, which intersects $A$ properly, such that $d\left(A_{i}\right)=2 k-1$.

This result suggests that "large" invariant differences $d\left(A_{i}\right)$ cannot occur too often. We exploit this observation more carefully and effectively as follows. Consider a finite increasing sequence $T=\left(x_{0}, x_{1}, \ldots, x_{t}\right)$ satisfying

$$
\frac{1}{2}=x_{0}<x_{1}<\ldots<x_{t}=k
$$

and the corresponding partition $I_{\text {fin }}=\bigcup_{m=1}^{t} K_{x_{m}}$, where

$$
K_{x_{m}}=\left\{i \in I_{\text {fin }} \mid \frac{k}{x_{m-1}}>d\left(A_{i}\right) \geq \frac{k}{x_{m}}\right\} .
$$

Then $K_{x_{m}}=J_{x_{m}} \backslash J_{x_{m-1}} \subseteq J_{x_{m}}$ and so $\left|K_{x_{m}}\right|<2 x_{m}$ by Lemma 3.8 and we have

$$
d\left(A_{K_{x_{m}}}\right)<2 x_{m} \cdot \frac{k}{x_{m-1}}=2 k \frac{x_{m}}{x_{m-1}} \text {. }
$$

Hence

$$
d\left(A_{\mathrm{fin}}\right)<2 k \cdot \min _{T} \sum_{m=1}^{t} \frac{x_{m}}{x_{m-1}},
$$

where the minimum is taken over all sequences $T$ satisfying (5). Our next task is to determine this minimum.

Lemma 3.10. $d\left(A_{\text {fin }}\right)<2 e k\lceil(\ln 2 k)\rceil$.

Proof. We prove that

$$
\min _{T} \sum_{m=1}^{t} \frac{x_{m}}{x_{m-1}} \leq\lceil(\ln 2 k)\rceil(2 k)^{\lceil(\ln 2 k)\rceil^{-1}} \leq e\lceil(\ln 2 k)\rceil,
$$

where the minimum is taken over all sequences $T$ as in (5). Denote $\delta_{m}=\frac{x_{m}}{x_{m-1}}$, $m=1, \ldots, t$. We must minimize the function

$$
S=S\left(t, \delta_{1}, \ldots, \delta_{t}\right)=\sum_{i=1}^{t} \delta_{i},
$$

given the conditions

$$
\prod_{i=1}^{t} \delta_{i}=\frac{x_{t}}{x_{0}}=2 k, \text { and } t \geq 1 .
$$

First observe that for a fixed integer $t$, the minimum of $S=S\left(\delta_{1}, \ldots, \delta_{t}\right)$ for $\delta_{1} \ldots \delta_{t}=2 k$ is attained at $\delta_{1}=\ldots=\delta_{t}=(2 k) \frac{1}{t}$ and is equal to $t(2 k) \frac{1}{t}$. 
(This question is equivalent to finding a $t$-dimensional parallelepiped of a given volume with minimal perimeter. The minimum is obtained by substituting $\delta_{t}=2 k /\left(\delta_{1} \ldots \delta_{t-1}\right)$ and then applying standard techniques from multivariable calculus to $S\left(\delta_{1}, \ldots, \delta_{t-1}\right)$.)

Now we have to find the minimum of the function $S(t)=t(2 k)^{\frac{1}{t}}$ of an integer variable $t$. Using standard techniques from calculus, we see that the function $S(x)=x(2 k)^{\frac{1}{x}}$ of the real variable $x$ attains its minimum at $x=$ $\ln 2 k$ and the minimum value is equal to $(\ln 2 k)(2 k)^{(\ln 2 k)^{-1}}=e(\ln 2 k)$. Thus our function $S(t)$ of the integer variable $t$ has minimum satisfying

$$
\min _{t \in \mathbb{Z}} S(t) \leq\lceil(\ln 2 k)\rceil(2 k)^{\lceil(\ln 2 k)\rceil^{-1}} \leq e\lceil(\ln 2 k)\rceil .
$$

In view of $(6), d\left(A_{\mathrm{fin}}\right)<2 e k\lceil(\ln 2 k)\rceil$.

Finally, we prove Theorem A.

Proof of Theorem A. In Lemmas 3.7-3.10 we used the fact that $A_{\text {fin }}$ is a (not necessarily finite) $k$-quasi-invariant subset. By Lemma 3.6, we can say more, namely that $A_{\text {fin }}$ is also $k^{\prime}$-quasi-invariant, where $k^{\prime}=k-d\left(A_{\text {inf }}\right)$. Thus, Lemma 3.10 should read as follows: $d\left(A_{\text {fin }}\right)<2 e k^{\prime}\left\lceil\left(\ln 2 k^{\prime}\right)\right\rceil$.

By Lemma 3.2, since $I_{\text {fin }}$ and $I_{\text {inf }}$ are finite by Lemmas 3.4 and 3.5, $d(A)=$ $d\left(A_{\text {fin }}\right)+d\left(A_{\text {inf }}\right)$, so

$$
d(A)<2 e\left(k-d\left(A_{\mathrm{inf}}\right)\right)\left\lceil\left(\ln 2 k^{\prime}\right)\right\rceil+d\left(A_{\mathrm{inf}}\right) \leq 2 e k\lceil(\ln 2 k)\rceil .
$$

\section{MORE BOUNDS}

We adopt here the notation from the previous section. First, we consider a general situation, which could be stated as follows. Given a $k$-quasi-invariant subset $A$, what can be said regarding the quasi-invariance of unions of some of its orbit segments? A possible answer is contained in the next result.

Proposition 4.1. Let $A$ be a disjoint union of $B_{1}$ and $B_{2}$, where $B_{i}$ is a union of some of the orbit segments of $A$, for $i=1,2$. Then

$$
k\left(B_{1}\right)+k\left(B_{2}\right) \leq 3 k / 2 \text {. }
$$

Proof. Set $x_{i}=k\left(B_{i}\right)$. Let $g_{i} \in G$ be such that $\left|B_{i} \backslash B_{i}^{g_{i}}\right|=x_{i}$ for $i=1,2$. By Lemma 3.1, $\left|B_{i}^{g_{i}} \backslash B_{i}\right|=x_{i}$, as well. There is nothing to prove if $x_{1}+x_{2} \leq k$, so we assume the contrary.

We claim that

$$
\left|B_{1}^{g_{1} g_{2}} \backslash B_{1}\right| \geq x_{1}+x_{2}-k .
$$

By Lemma 3.1, $\left|B_{1} \backslash B_{1}^{g_{2}}\right|=\left|B_{1}^{g_{2}} \backslash B_{1}\right| \leq k-x_{2}$. Set $C=B_{1}^{g_{1}} \backslash B_{1},|C|=x_{1}$. Note that $C^{g_{2}} \cap B_{1} \subseteq B_{1} \backslash B_{1}^{g_{2}}$, so $\left|C^{g_{2}} \cap B_{1}\right| \leq\left|B_{1} \backslash B_{1}^{g_{2}}\right| \leq k-x_{2}$. Hence $\left|C^{g_{2}} \backslash B_{1}\right| \geq x_{1}-\left(k-x_{2}\right)$. But

$$
C^{g_{2}} \backslash B_{1}=\left(B_{1}^{g_{1} g_{2}} \backslash B_{1}^{g_{2}}\right) \backslash B_{1} \subseteq B_{1}^{g_{1} g_{2}} \backslash B_{1},
$$

whence (7) follows.

Replace in the above argument $B_{1}$ with $B_{2}, g_{2}$ with $g_{1}^{-1}$, and $g_{1}$ with $g_{2}^{-1}$. The argument remains valid since $\left|B_{i}^{g_{j}^{-1}} \backslash B_{i}\right|=\left|B_{i} \backslash B_{i}^{g_{j}}\right|$ and, by Lemma 3.1, $\left|B_{i} \backslash B_{i}^{g_{j}}\right|=\left|B_{i}^{g_{j}} \backslash B_{i}\right|$, for $i, j=1,2$. We obtain, similarly to (7), that

$$
\left|B_{2}^{g_{2}^{-1} g_{1}^{-1}}\right| B_{2} \mid \geq x_{1}+x_{2}-k \text {. }
$$


Applying Lemma 3.1 again, we have

$$
\left|B_{2}^{g_{1} g_{2}} \backslash B_{2}\right|=\left|\left(B_{2}^{g_{2}^{-1} g_{1}^{-1}} \backslash B_{2}\right)^{g_{1} g_{2}}\right| \geq x_{1}+x_{2}-k \text {. }
$$

Therefore

$$
k \geq\left|A^{g_{1} g_{2}} \backslash A\right|=\left|B_{1}^{g_{1} g_{2}} \backslash B_{1}\right|+\left|B_{2}^{g_{1} g_{2}} \backslash B_{2}\right| \geq 2\left(x_{1}+x_{2}-k\right),
$$

that is, $3 k \geq 2\left(x_{1}+x_{2}\right)$.

The bound can be attained when $k$ is a power of 2 . Let $A$ and $G$ be as in the remark following Theorem B, that is, $G$ is an elementary abelian 2group of rank $r$ acting on the disjoint union of coset spaces of all subgroups of $G$ of index 2 , and $A$ meets each orbit in a point. Let $B_{1}$ be the set of fixed points of some non-identity element of $G$, and $B_{2}$ be its complement in $A$. It is straightforward to check that $k\left(B_{1}\right)=k / 2, k\left(B_{2}\right)=k$, that is, $k\left(B_{1}\right)+k\left(B_{2}\right)=3 k / 2$.

Proof of Theorem C. Set $k=k(A)$. We proceed by induction on $m=m(A)$. For $m=1$ the statement is just Corollary 2.3, so suppose $m \geq 2$. Assume that the result holds for all $m \leq m_{0}$, when $m_{0}$ is a power of 2 , that is,

$$
d(A) \leq 2 k(A)(3 / 2)^{\left\lceil\log _{2} m_{0}\right\rceil}-2^{\left\lceil\log _{2} m_{0}\right\rceil} .
$$

We show that it holds for all $m$ such that $2 m_{0} \geq m>m_{0}$. Divide $A$ into two parts $B_{1}$ and $B_{2}$, as in Proposition 4.1, such that $m\left(B_{i}\right) \leq m_{0}$, for $i=1,2$, and set $k_{i}=k\left(B_{i}\right)$. By the inductive hypothesis, (8) holds for $d\left(B_{i}\right)$ for $i=1,2$. By Proposition 4.1, $k_{2} \leq 3 k / 2-k_{1}$. Thus

$$
\begin{aligned}
d(A) & =d\left(B_{1}\right)+d\left(B_{2}\right) \\
& \leq 2 k_{1}(3 / 2)^{\left\lceil\log _{2} m_{0}\right\rceil}-2^{\left\lceil\log _{2} m_{0}\right\rceil}+2\left(3 k / 2-k_{1}\right)(3 / 2)^{\left\lceil\log _{2} m_{0}\right\rceil}-2^{\left\lceil\log _{2} m_{0}\right\rceil} \\
& =2 k(3 / 2)^{\left\lceil\log _{2} 2 m_{0}\right\rceil}-2^{\left\lceil\log _{2} 2 m_{0}\right\rceil}=2 k(3 / 2)^{\left\lceil\log _{2} m\right\rceil}-2^{\left\lceil\log _{2} m\right\rceil} \cdot \quad \square
\end{aligned}
$$

\section{ACKNOWLEDGMENT}

The authors wish to thank Peter M. Neumann for his interest and many valuable comments.

\section{REFERENCES}

1. G.M. Bergman and H.W. Lenstra, Jr., Subgroups close to normal subgroups, J. Algebra 127 (1989), 80-97.

2. B.J. Birch, R.G. Burns, S. Oates Macdonald, and P.M. Neumann, On the orbit-sizes of permutation groups containing elements separating finite subsets, Bull. Austral. Math. Soc. 14 (1976), 7-10.

3. L. Brailovsky, Structure of quasi-invariant sets, Arch. Math. (Basel) 59 (1992), 322-326.

4. L. Brailovsky, D.V. Pasechnik, and C.E. Praeger, Classification of 2-quasi-invariant sets, Ars Combin. (to appear).

5. P. Dembowski, Finite geometries, Springer-Verlag, New York, 1968.

6. P. Erdös, C. Ko, and R. Rado, Intersection theorems for systems of finite sets, Quart. J. Math. Oxford Ser. (2) 12 (1961), 313-320.

7. P. Frankl and Z. Füredi, Non-trivial intersecting families, J. Combin. Theory Ser. A 41 (1986), 150-153. 
8. B.H. Neumann, Groups covered by permutable subsets, J. London Math. Soc. (2) 29 (1954), 236-248.

9. C.E. Praeger, On permutation groups with bounded movement, J. Algebra 44 (1991), 436-442.

Department of Mathematics, University of Western Australia, Nedlands, Perth, Western Australia 6009, Australia

Current address, L. Brailovsky: Scitex Corporation Ltd., P. O. Box 330, Herzliya, 66103, Israel E-mail address, L. Brailovsky: leonid@mhs . scitex.com

E-mail address, D. V. Pasechnik: pasececan.nl

E-mail address, C. E. Praeger: praeger@maths uwa.oz .au 